\title{
Non-Laplacian Beliefs in a Global Game with Noisy Signaling*
}

\author{
Chris Edmond ${ }^{\dagger}$
}

First draft: August 2012. This draft July 2017

\begin{abstract}
In standard global games, individual behavior is optimal if it constitutes a best response to agnostic — Laplacian — beliefs about the aggregate behavior of other agents. This paper considers a standard binary action global game augmented with noisy signaling by an informed policy-maker and shows that in this game, equilibrium beliefs depart in quite stark ways from the Laplacian benchmark. In the limit as signals become arbitrarily precise, so that all fundamental uncertainty is removed (leaving only strategic uncertainty), the equilibrium beliefs of the marginal individual concerning the aggregate action collapse to a discrete Bernoulli distribution, giving probability mass only to the polar extreme outcomes. By contrast in the underlying standard global game the marginal individual believes the aggregate action has a continuous uniform distribution, giving equal likelihood to all possible outcomes.
\end{abstract}

Keywords: coordination, signaling, bias, strategic uncertainty, noise.

JEL classifications: C7, D7, D8.

* Some of this material previously circulated in abbreviated form in the appendix to NBER Working Paper 17395, September 2011. I wrote this short paper because I thought these results too intriguing to leave buried in a technical appendix. I thank Claudio Mezzetti for helpful comments. Vivianne Vilar provided excellent research assistance.

$\dagger$ University of Melbourne. Email: 〈cedmond@unimelb.edu.au〉. 


\section{Introduction}

Many economic phenomena can be analyzed as coordination games. ${ }^{1}$ The complete information versions of coordination games frequently feature multiple equilibria, but in a pioneering contribution Carlsson and van Damme (1993) showed that a unique equilibrium can often be selected by first considering a family of incomplete information games indexed by the amount of noise in players' signals and then taking the limit as the signal noise is driven to zero. This equilibrium selection is attractive, not least because it makes for sharper conclusions about how equilibrium outcomes vary with parameters of interest, and has helped make the resulting global games methodology a very popular modeling tool. ${ }^{2}$

Perhaps surprisingly, optimal behavior in these coordination games with incomplete information generally does not require agents to use subtle probabilistic reasoning. Rather, the opposite is more true: standard global games feature the striking property that each individual will be behaving optimally if their behavior constitutes a best response to beliefs that are agnostic as to the aggregate behavior of others. In this sense, individuals will be acting reasonably even if they have quite naive beliefs. Morris and Shin (2003) refer to these as Laplacian beliefs in reference to Pierre Simon Laplace's early use of the uniform prior. ${ }^{3}$ This paper considers a signaling extension of a standard global game, introduced by Edmond (2013), and shows that while equilibrium actions are similar to those in the underlying game, equilibrium beliefs involve dramatic departures from the Laplacian benchmark.

To fix ideas, consider a standard symmetric binary action global game. There is an unobserved state variable $\theta$. A continuum of players begin with common uninformative priors and then receive idiosyncratic signals $x_{i}$ centered on $\theta$. Each player can take a risky action $a_{i}=1$ or a safe action $a_{i}=0$. The risky action pays off if and only if the proportion of individuals taking the risky action, $A \in[0,1]$, is larger than $\theta$. Individual actions $a_{i}$ and the aggregate action $A$ are strategic complements. As is well known from Carlsson and van Damme (1993) and Morris and Shin (1998), this game has a unique equilibrium and the equilibrium is characterized by a critical signal $x^{*}$ such that individuals with signals $x_{i}<x^{*}$ take the risky action. This standard global game features the property that each individual views themselves as typical in a particularly strong sense. Each individual believes the proportion of individuals with signals $x \leq x_{i}$ is uniformly distributed on the interval $[0,1]$. In this sense individuals are agnostic as to their rank-order in the population. The intuition for this is that when individuals begin with no prior information on the state $\theta$ their signal $x_{i}$ anchors their expectation about $\theta$ but provides no information about the

\footnotetext{
${ }^{1}$ For example, Diamond and Dybvig $(1983)$ on bank runs, Obstfeld $(1986,1996)$ on currency crises, Calvo (1988) and Cole and Kehoe (2000) on debt crises, and Katz and Shapiro (1986) on technology adoption.

${ }^{2}$ Prominent examples include Morris and Shin (1998) on currency crises, Chamley (1999) and Dasgupta (2007) on investment complementarities, and Rochet and Vives (2004) and Goldstein and Pauzner (2005) on bank runs. For overviews, see Morris and Shin (2000, 2003) or Vives (2005).

${ }^{3}$ And latter somewhat facetiously referred to as the "principle of insufficient reason," or in the terminology of Keynes (1921), the "principle of indifference". See Stigler (1986) for further discussion.
} 
proportion of individuals who observe higher (or lower) signals. Since the aggregate action $A$ is the proportion of individuals with signals less than the critical $x^{*}$, a special case of this is that the marginal individual with signal $x_{i}=x^{*}$ believes the aggregate action $A$ is likewise uniformly distributed on $[0,1]$. Thus, in this standard global game, individuals will be behaving optimally if they simply choose the action $a_{i}$ that is a best response to the naive belief that $A$ is uniformly distributed on $[0,1]$.

To this standard global game I add a policy-maker who is informed about $\theta$ and who seeks to induce coordination on the status quo. The policy-maker is endowed with the ability to choose the mean of the distribution from which individuals draw signals. The cost of choosing a signal mean $y$ is increasing in the amount of bias $y-\theta$ the policy-maker introduces. Along the equilibrium path, individuals receive signals of the form $x_{i}=y(\theta)+\varepsilon_{i}$ where $\varepsilon_{i}$ is idiosyncratic noise. In this sense, the policy-maker can engage in noisy signaling. In a companion paper, Edmond (2013), I study this setup at length and show that this game has a unique perfect Bayesian equilibrium, despite the endogenous information structure. ${ }^{4}$

The main contribution of the present paper is to show that the global game with noisy signaling ${ }^{5}$ implies equilibrium beliefs that are qualitatively completely distinct from those in the underlying standard global game. To be clear, this model is not the only example of a global game with non-Laplacian beliefs - see, e.g., Corsetti, Dasgupta, Morris and Shin (2004) and Dasgupta (2007) for other examples — but, to the best of my knowledge, this is the first paper to give a complete analytical characterization of the ways in which beliefs fail to be Laplacian in this kind of setting and the first to show just how different the equilibrium beliefs turn out to be. In particular, Proposition 1 shows that individual beliefs about the proportion of individuals with signals $x \leq x_{i}$, i.e., about their rank-order in the population, are far from uniform. The equilibrium beliefs feature both an atom (a discrete amount of probability mass concentrated on a single point) and a gap in the support. These features follow from the policy-maker's equilibrium signaling policy $y(\theta)$ which allows an interval of types $\theta \in\left(\theta^{*}, \theta^{* *}\right)$ to pool on a common signal mean, $y(\theta)=y^{*}$ say. This pooling redistributes probability mass from outcomes corresponding to the interval $\left(\theta^{*}, \theta^{* *}\right)$ and concentrates it on a single point corresponding to $\theta^{*}$ thereby creating a gap in the support of the rank-order beliefs and creating an atom at a point corresponding to $\theta^{*}$. Proposition 2 shows that the marginal individual with signal $x_{i}=x^{*}$ likewise has beliefs about the aggregate action $A$ that are far from uniform and provides an explicit characterization of those beliefs in terms of model parameters. Finally, Proposition 3 considers the limit as

\footnotetext{
${ }^{4}$ This contrasts with Angeletos, Hellwig and Pavan (2006), who were the first to consider signaling in global games. The key reason for the uniqueness in my model relative to theirs is that in my setting individuals' endogenous signals are a monotone function of the state whereas in Angeletos, Hellwig and Pavan (2006) the endogenous signals are a non-monotone function of the state that gives individual information receivers more common belief as to whether the status quo is likely to prevail or not. The greater amount of common belief in their setting makes it easier to sustain multiple equilibria. See Edmond (2013) for further discussion.

${ }^{5}$ Other global games with endogenous information include Angeletos and Werning (2006), Hellwig, Mukherji and Tsyvinski (2006), Angeletos, Hellwig and Pavan (2007), and Dasgupta (2007).
} 
the signal precision becomes arbitrarily high, so that all fundamental uncertainty is removed (leaving only strategic uncertainty). In this limit, the marginal individual with signal $x^{*}$ believes the aggregate action $A$ has the discrete Bernoulli distribution on the polar extreme outcomes $\{0,1\}$, not a uniform distribution on the interval $[0,1]$. Instead of assigning equal likelihood to every possible outcome, in this limit the marginal individual concentrates all belief on the extremes. In short, the equilibrium beliefs are not only non-Laplacian, they are about as far from Laplacian as one could imagine them to be. As a result, and unlike in a standard global game, an individual who chooses an action $a_{i}$ that is a best response to the naive belief that $A$ is uniformly distributed on $[0,1]$ will generally be behaving sub-optimally.

The main significance of these non-Laplacian beliefs is that optimal play in the model with noisy signaling requires agents to use subtle probabilistic reasoning that is simply unnecessary in a standard global game. ${ }^{6}$ In turn, this suggests the Laplacian property is less an intrinsic property of this class of games and rather more a consequence of the relatively simple information structures that are generally used in standard global games applications.

Section 2 briefly presents the model and characterizes the equilibrium with noisy signaling. Section 3 reviews the Laplacian property of beliefs in standard global games. Section 4 is the heart of the paper and provides all the main results on non-Laplacian beliefs in the equilibrium with noisy signaling. Section 5 concludes. All proofs are in the Appendix.

\section{Model}

The model follows Edmond (2013) closely. There is a unit mass of ex ante identical individuals, indexed by $i \in[0,1]$, facing a collective action problem. Each individual decides whether to take a safe action $a_{i}=0$ or a risky action $a_{i}=1$. The aggregate action is $A:=\int_{0}^{1} a_{i} d i$.

Individual payoffs. Taking the risky action $a_{i}=1$ incurs a private opportunity cost $p \in(0,1)$ and delivers a payoff of 1 if and only if the aggregate action $A$ is sufficiently high. In particular, there is an imperfectly observed state variable $\theta$ such that the status quo prevails unless $A>\theta$. A high value of $\theta$ means that the status quo is more likely to prevail. Individual payoffs can be written

$$
u\left(a_{i}, A, \theta\right):=(\mathbb{1}\{A>\theta\}-p) a_{i}
$$

where $\mathbb{1}\{\cdot\}$ denotes the indicator function. An individual will take the risky action, $a_{i}=1$, whenever they expect the event $\{A>\theta\}$ with more than probability $p$. Individual actions

\footnotetext{
${ }^{6}$ As stressed by Morris and Shin (2003), one of the attractive features of the Laplacian property in standard global games is the implication that equilibria can be sustained without requiring superhuman degrees of rationality (and in this sense, such equilibria can be considered relatively 'robust').
} 
$a_{i}$ and the aggregate action $A$ are strategic complements: the more individuals take the risky action, the more likely it is that any individual's best response is to also take the risky action.

Informed policy maker. There is a single policy-maker with a vested interest in preserving the status quo. For example, this agent can be interpreted as an autocratic regime seeking to prevent a revolt, or as the monopolist supplier of an existing technology platform that is threatened by the possibility of consumers switching to an alternative platform. Importantly, the policy-maker is better informed about the difficulty of achieving coordination than are the individuals. In particular, the exact value of $\theta$ is known to the policy-maker. The policymaker obtains a gross benefit $\theta-A$ so long as the status quo prevails and obtains an outside option normalized to zero otherwise. ${ }^{7}$

Costly noisy signaling. Individuals begin with common priors for $\theta$, specifically the improper uniform on $\mathbb{R}$. They then observe an idiosyncratic signal $x_{i}:=y+\varepsilon_{i}$ where the noise $\varepsilon_{i}$ is IID normal across individuals, independent of $\theta$, and has mean zero and precision $\alpha>0$ (i.e., variance $1 / \alpha$ ). Thus, conditional on $y$ the signal density is

$$
f\left(x_{i} \mid y\right):=\sqrt{\alpha} \phi\left(\sqrt{\alpha}\left(x_{i}-y\right)\right)
$$

where $\phi(\cdot)$ denotes the standard normal PDF.

To defend the status quo, the policy-maker can choose the signal mean $y$ at a cost $c(y-\theta)$ with constant marginal cost $c>0 .{ }^{8}$ The term $b:=y-\theta$ is a measure of the bias that the policy-maker is trying to inject into individual signals. Given this bias, individual signals are of the form $x_{i}=\theta+b+\varepsilon_{i}$. If $b>0$ individuals are receiving signals that, taken at face value, suggest it will be hard to overturn the status quo. Choosing $b>0$ incurs a positive cost. The policy-maker can always avoid this cost by choosing $b=0$.

The net payoff to the policy-maker can be written

$$
B(\theta, A)-c(y-\theta)=\max [0, \theta-A]-c(y-\theta)
$$

If $\theta<A$, the policy maker will always choose $y=\theta$ and thereby obtain a net payoff of zero.

Equilibrium. A symmetric perfect Bayesian equilibrium of this model consists of individual beliefs $\pi\left(\theta \mid x_{i}\right)$ and individual actions $a\left(x_{i}\right)$, an aggregate action $A(y)$, and policy-maker's bias $b(\theta)$ such that: (i) an individual with signal $x_{i}$ rationally takes into account the bias

\footnotetext{
${ }^{7}$ Some interpretations of the policy-maker require reversing the preference ordering. For example, an entering firm seeking to persuade customers to switch to its new platform would want to maximize $A-\theta$. The analysis below also holds for this interpretation once the sign of $\theta$ and the direction of the policy-maker's preferred bias are switched.

${ }^{8}$ Edmond (2013) considers more general convex costs. Here I specialize to the case of constant marginal costs because this permits an exact analytic characterization of equilibrium beliefs that are of principle interest in this paper.
} 
$y(\theta)=\theta+b(\theta)$ when forming their posterior beliefs, (ii) given these beliefs $a\left(x_{i}\right)$ maximizes individual expected utility, (iii) the aggregate action is consistent with the individual actions, and (iv) the bias $b(\theta)$ maximizes the policy-maker's payoff given the aggregate action. In equilibrium, the status quo is overturned if $\theta<A(y(\theta))$ and otherwise prevails.

\subsection{Standard global game}

If it is common knowledge that no bias will occur (e.g., $c=+\infty$ ) so that $b(\theta)=0$ for all $\theta$, then each individual simply has signal $x_{i}=\theta+\varepsilon_{i}$ and the analysis reduces to a standard symmetric binary action global game. Following arguments familiar from Carlsson and van Damme (1993) and Morris and Shin (1998), this game has a unique equilibrium. The equilibrium is characterized by two thresholds, a critical $\theta_{\mathrm{MS}}^{*}$ such that the status quo is overturned for $\theta<\theta_{\mathrm{MS}}^{*}$ and a critical signal $x_{\mathrm{MS}}^{*}$ such that an individual takes the risky action for $x_{i}<x_{\mathrm{MS}}^{*}$. These thresholds are determined as follows:

Morris-Shin Benchmark. The equilibrium thresholds $x_{\mathrm{MS}}^{*}, \theta_{\mathrm{MS}}^{*}$ simultaneously solve

$$
\begin{gathered}
\operatorname{Prob}\left[\theta<\theta_{\mathrm{MS}}^{*} \mid x_{\mathrm{MS}}^{*}\right]=\Phi\left(\sqrt{\alpha}\left(\theta_{\mathrm{MS}}^{*}-x_{\mathrm{MS}}^{*}\right)\right)=p \\
\operatorname{Prob}\left[x_{i}<x_{\mathrm{MS}}^{*} \mid \theta_{\mathrm{MS}}^{*}\right]=\Phi\left(\sqrt{\alpha}\left(x_{\mathrm{MS}}^{*}-\theta_{\mathrm{MS}}^{*}\right)\right)=\theta_{\mathrm{MS}}^{*}
\end{gathered}
$$

where $\Phi(\cdot)$ denotes the standard normal CDF and $p$ is the opportunity cost of taking the risky action. In particular, $\theta_{\mathrm{MS}}^{*}=1-p$, independent of $\alpha$, and $x_{\mathrm{MS}}^{*}=1-p-\Phi^{-1}(p) / \sqrt{\alpha}$.

Briefly, the first condition says that if the policy-maker's threshold is $\theta_{\mathrm{MS}}^{*}$, the marginal individual with signal $x_{i}=x_{\mathrm{MS}}^{*}$ expects the status quo to be overturned with exactly probability $p$. The second says that if the signal threshold is $x_{\mathrm{MS}}^{*}$, a policy-maker with $\theta=\theta_{\mathrm{MS}}^{*}$ will be indifferent to the status quo being overturned or not. I say that the policy-maker's noisy signaling is effective if in equilibrium $\theta^{*}<\theta_{\mathrm{MS}}^{*}=1-p$.

\subsection{Equilibrium with noisy signaling}

With noisy signaling, the information structure is endogenous and, in equilibrium, the beliefs of individuals and the policy-maker's incentives to introduce bias have to be rendered mutually consistent. Despite the endogenous information, however, it can be shown that this game still has a unique equilibrium and that the equilibrium can be characterized in a similar way to the benchmark global game with exogenous information. The equilibrium is characterized by two thresholds $x^{*}, \theta^{*}$ and a function $y(\theta)=\theta+b(\theta)$ giving the equilibrium signal mean (and hence the equilibrium amount of bias).

Here I simply provide the conditions that have to be satisfied in equilibrium. The interested reader should consult Edmond (2013) for more details and the proof that this is indeed the only equilibrium. 
Policy-maker's problem. In equilibrium, individuals take the risky action $a\left(x_{i}\right)=1$ for $x_{i}<x^{*}$, so the aggregate action facing a policy maker that has chosen signal mean $y$ is

$$
A(y)=\Phi\left(\sqrt{\alpha}\left(x^{*}-y\right)\right)
$$

The status quo is overturned for $\theta<\theta^{*}$ and, given this, policy-makers with low $\theta$ never introduce bias, i.e., $y(\theta)=\theta$ for $\theta<\theta^{*}$, since otherwise they would be incurring a cost but receiving no benefit. For all $\theta \geq \theta^{*}$, the policy-maker choses

$$
y(\theta) \in \underset{y \geq \theta}{\operatorname{argmin}}\left[\Phi\left(\sqrt{\alpha}\left(x^{*}-y\right)\right)+c(y-\theta)\right]
$$

The critical $\theta^{*}$ is then determined by the boundary condition

$$
\theta^{*}=\Phi\left(\sqrt{\alpha}\left(x^{*}-y\left(\theta^{*}\right)\right)\right)+c\left(y\left(\theta^{*}\right)-\theta^{*}\right)
$$

The marginal policy-maker $\theta^{*}$ is indifferent to the status quo being overturned or not. For any given signal threshold $x^{*}$, equations (6)-(7) determine $\theta^{*}$ and the signaling policy $y(\theta)$.

Information receiver's problem. An individual takes the risky action $a\left(x_{i}\right)=1$ only if they believe the status quo will be overturned with probability at least $p$. Using Bayes's Rule, the marginal individual with signal $x_{i}=x^{*}$ is indifferent when

$$
\operatorname{Prob}\left[\theta<\theta^{*} \mid x^{*}, y(\cdot)\right]=\frac{\int_{-\infty}^{\theta^{*}} \sqrt{\alpha} \phi\left(\sqrt{\alpha}\left(x^{*}-\theta\right)\right) d \theta}{\int_{-\infty}^{\infty} \sqrt{\alpha} \phi\left(\sqrt{\alpha}\left(x^{*}-y\left(\theta^{\prime}\right)\right) d \theta^{\prime}\right.}=p
$$

where the numerator uses $y(\theta)=\theta$ for all $\theta<\theta^{*}$ and where it should be borne in mind that the signaling policy $y(\theta)$ also depends on $x^{*}$. An equilibrium consists of a $x^{*}, \theta^{*}$ and $y(\theta)$ function simultaneously solving (6), (7) and (8). In Edmond (2013) I show that there is a unique equilibrium and characterize its properties in detail.

Signaling policy. Consider a policy-maker facing aggregate action $A(y)$. If the choice of $y$ is to induce continuation of the status quo it must minimize $A(y)+c y$ subject to the constraint $y \geq \theta$. Since the first-order condition for interior solutions is $-A^{\prime}(y)=c$, all policy-makers with interior solutions choose the same signal mean, $y^{*}$ say. Corner solutions can arise for two reasons: (i) for all $\theta$ such that $\theta<A\left(y^{*}\right)+c\left(y^{*}-\theta\right)$ the status quo is a hopeless cause and the policy-maker finds it better to give way rather than pay the cost of taking $y^{*}$, and (ii) for all $\theta \geq y^{*}$ the status quo is sufficiently locked-in that there is no need to undertake the cost of $y^{*}$ to defend it (i.e., the non-negativity constraint $y \geq \theta$ is binding). All policy-makers at a corner have $y(\theta)=\theta$ so that there is no bias. All policy-makers that are at an interior solution have $y(\theta)=y^{*}$ with bias $b(\theta)=y^{*}-\theta$. In short,

$$
y(\theta)= \begin{cases}\theta & \text { if } \theta \notin\left[\theta^{*}, \theta^{* *}\right) \\ y^{*} & \text { if } \theta \in\left[\theta^{*}, \theta^{* *}\right)\end{cases}
$$


The critical point $\theta^{*}$ is implicitly determined by the indifference condition $\theta^{*}=A\left(y^{*}\right)+$ $c\left(y^{*}-\theta^{*}\right)$ as above. The higher critical point $\theta^{* *}$ is simply equal to the constant $y^{*}$, since it is the smallest policy-maker type for which the non-negativity constraint $y^{*} \geq \theta$ is binding. Figure 1 illustrates. $^{9}$

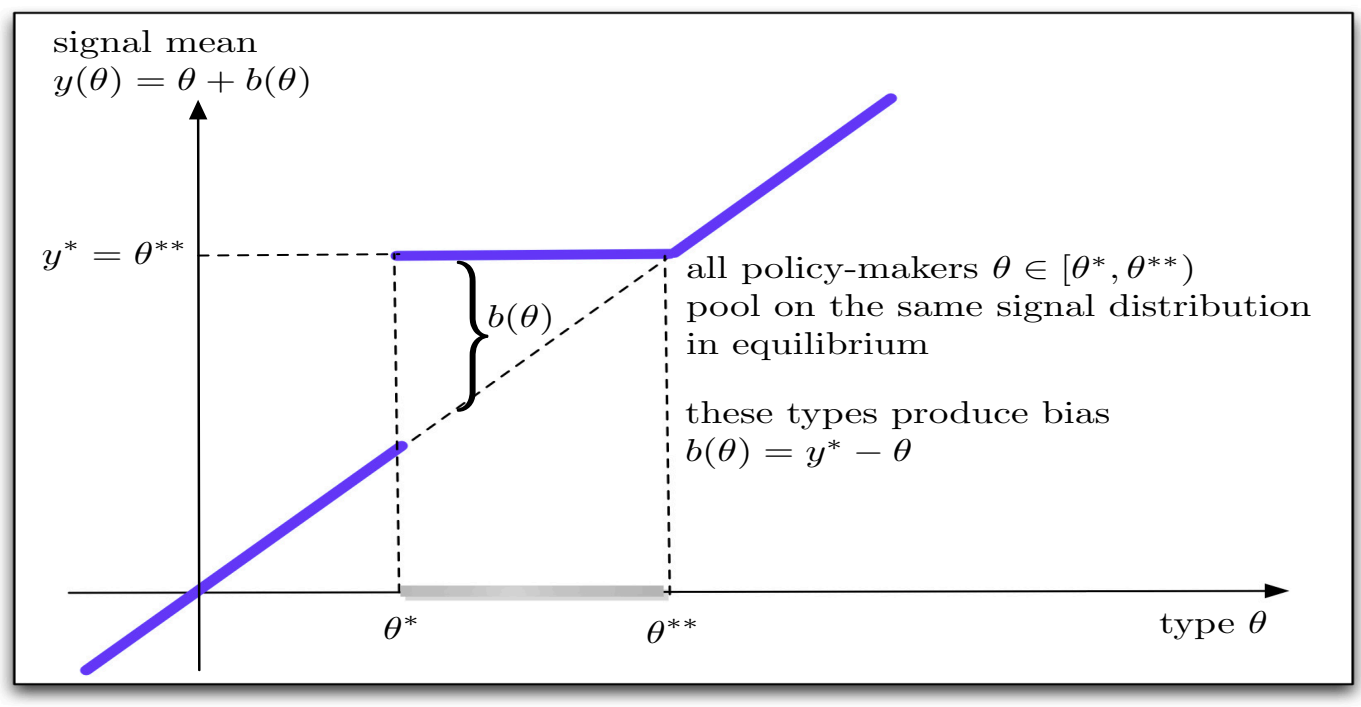

Figure 1: Signal mean and signal bias.

Signal mean $y(\theta)=\theta+b(\theta)$. The status quo is overturned for all $\theta<\theta^{*}$. All policy-makers with $\theta \in\left[\theta^{*}, \theta^{* *}\right)$ choose the same signal mean $y^{*}=\theta^{* *}$ and thus generate the same signal distribution in equilibrium. The bias $b(\theta)=y^{*}-\theta$ allows them to each mimic a strong type $\theta^{* *}$, i.e., the lowest type such that the status quo prevails without costly intervention. All these types generate signals $x_{i}=y^{*}+\varepsilon_{i}$ that are (locally) uninformative about $\theta$.

Signaling effective when $\alpha$ sufficiently high. A key property of the equilibrium is that the policy-maker's signaling is effective, in the sense of reducing the threshold $\theta^{*}$ below the Morris-Shin benchmark $\theta_{\mathrm{MS}}^{*}=1-p$, when the signal precision is sufficiently high. In particular, Edmond (2013) proves the following result, used extensively below:

LEMma 1. In the limit as the signal precision $\alpha \rightarrow+\infty$, the critical threshold $\theta^{*} \rightarrow 0^{+}$.

Thus when the signal precision is sufficiently high, equilibrium outcomes break decisively in the policy-maker's favor. In the limit, all types $\theta \geq 0$ that can survive, do survive. While interesting in its own right, this result also turns out to have crucial implications for equilibrium beliefs in the limit as fundamental uncertainty vanishes.

\footnotetext{
${ }^{9}$ For a general convex cost function we would still have a jump at $\theta^{*}$, but having an interval on which $y(\theta)$ is constant is special to the linear cost function used here. For a strictly convex cost function, $y(\theta)$ is strictly increasing in $\theta$ for all $\theta>\theta^{*}$. The qualitative features of the strictly convex case are, however, similar to those of the linear case when the signal precision $\alpha$ is large, which is the case of interest in this paper.
} 


\section{Laplacian beliefs in standard global game}

In the absence of noisy signaling, the model reduces to a standard global game. In this game, individuals believe themselves to be typical in a particularly strong sense. Intuitively, since individuals have uninformative priors for $\theta$, their signal $x_{i}$ contains no information about their rank-order in the population and thus provides them with no information about the proportion of individuals who observe higher (or lower) signals. This agnosticism about their rank-order translates into a similar agnosticism about the size of the aggregate action.

Rank-order beliefs. The following discussion draws heavily on Morris and Shin (2007). Let $l(x, \theta) \in[0,1]$ denote the proportion of individuals with signals below $x$ if the true state is $\theta$, that is

$$
l(x, \theta):=\operatorname{Prob}\left[x_{j} \leq x \mid \theta\right]
$$

This is a function of the random variable $\theta$ and individual beliefs about this proportion depend on their beliefs about $\theta$. Now consider an individual's beliefs about their own rankorder in the population. That is, fix an individual with signal $x_{i}$ and let $G\left(k \mid x_{i}\right)$ denote the CDF of $l\left(x_{i}, \theta\right)$ conditional on their own $x_{i}$. Specifically,

$$
G\left(k \mid x_{i}\right):=\operatorname{Prob}\left[l\left(x_{i}, \theta\right) \leq k \mid x_{i}\right]
$$

In this standard global game individuals have signals $x_{i}=\theta+\varepsilon_{i}$ where the noise $\varepsilon_{i}$ is IID normal with mean zero and precision $\alpha$. So, conditional on $\theta$, the proportion of individuals $l\left(x_{i}, \theta\right)$ with signals below $x_{i}$ is

$$
l\left(x_{i}, \theta\right):=\operatorname{Prob}\left[x_{j} \leq x_{i} \mid \theta\right]=\Phi\left(\sqrt{\alpha}\left(x_{i}-\theta\right)\right)
$$

Equivalently, $l\left(x_{i}, \theta\right) \leq k$ if and only if $\theta \geq x_{i}-\Phi^{-1}(k) / \sqrt{\alpha}$. The beliefs of an individual $x_{i}$ about their rank order in the population are then given by

$$
G\left(k \mid x_{i}\right):=\operatorname{Prob}\left[l\left(x_{i}, \theta\right) \leq k \mid x_{i}\right]=\operatorname{Prob}\left[\theta \geq x_{i}-\frac{1}{\sqrt{\alpha}} \Phi^{-1}(k) \mid x_{i}\right]
$$

and since each individual's posterior beliefs for $\theta$ are likewise normal with mean $x_{i}$ and precision $\alpha$, this evaluates to ${ }^{10}$

$$
\begin{aligned}
G\left(k \mid x_{i}\right) & =\int_{x_{i}-\Phi^{-1}(k) / \sqrt{\alpha}}^{\infty} \sqrt{\alpha} \phi\left(\sqrt{\alpha}\left(\theta-x_{i}\right)\right) d \theta \\
& =1-\Phi\left(\sqrt{\alpha}\left(x_{i}-\frac{1}{\sqrt{\alpha}} \Phi^{-1}(k)-x_{i}\right)\right) \\
& =1-\Phi\left(-\Phi^{-1}(k)\right) \\
& =k
\end{aligned}
$$

\footnotetext{
${ }^{10}$ More generally (e.g., in the case of informative priors), the following calculation holds in the limit as $\alpha \rightarrow \infty$. This will be the situation of primary interest below. See Hellwig (2002) and Morris and Shin (2000, 2003) for further discussion of the importance of informative priors in standard global games.
} 
These rank-order beliefs $G\left(k \mid x_{i}\right)=k$ have two key properties: (i) they are independent of $x_{i}$, an individual's signal $x_{i}$ is uninformative about their rank-order in the population, and (ii) they are uniform in $k$, each individual believes that the proportion of individuals $l\left(x_{i}, \theta\right)$ with signals less than their own is uniformly distributed on $[0,1]$. These are conceptually distinct properties in that, in principle, an individual could have rank-order beliefs that depend on $x_{i}$ but are nonetheless uniform in $k$ (if the support of the rank-order beliefs depends on $x_{i}$, for example). Taken together, these two properties imply that each individual considers themselves to be typical and makes them agnostic as to their rank-order in the population.

Beliefs about the aggregate action. In this standard global game, the rank-order beliefs translate directly into beliefs about the size of the aggregate action $A(\theta)$. In particular, recall that in equilibrium an individual $j$ takes the risky action $a_{j}=1$ if and only if their signal is $x_{j}<x^{*}$. Thus the aggregate action is

$$
A(\theta)=\operatorname{Prob}\left[x_{j} \leq x^{*} \mid \theta\right]
$$

But this is just the rank-order function $l(x, \theta)$ evaluated at the marginal individual with signal $x=x^{*}$, that is

$$
A(\theta)=\operatorname{Prob}\left[x_{j} \leq x^{*} \mid \theta\right]=l\left(x^{*}, \theta\right)=\Phi\left(\sqrt{\alpha}\left(x^{*}-\theta\right)\right)
$$

Now consider the beliefs of an arbitrary individual with signal $x_{i}$ considering taking the risky action. Following the same steps as above, it is straightforward to show that the CDF for the event $\{A(\theta) \leq k\}$ is simply

$$
\operatorname{Prob}\left[A(\theta) \leq k \mid x_{i}\right]=\Phi\left(\sqrt{\alpha}\left(x_{i}-x^{*}+\frac{1}{\sqrt{\alpha}} \Phi^{-1}(k)\right)\right)
$$

And when evaluated at $x_{i}=x^{*}$ this reduces to $\operatorname{Prob}\left[A(\theta) \leq k \mid x^{*}\right]=k$ as above. Since every individual has uniform rank-order beliefs and since the size of the aggregate action is simply the proportion of individuals with signals less than the marginal $x^{*}$, the marginal individual likewise believes the aggregate action $A(\theta)$ is uniformly distributed on $[0,1]$. The identity of the marginal individual depends, of course, on the parameters of the game. For example, in the standard global game, $x^{*}=1-p-\Phi^{-1}(p) / \sqrt{\alpha}$. But whoever the marginal individual is, they believe the aggregate action is uniformly distributed on $[0,1]$. Put differently, the marginal individual is precisely that individual who, if equipped with uniform beliefs about $A(\theta)$, would be indifferent between taking the risky action or not.

\section{Non-Laplacian beliefs in noisy signaling equilibrium}

I now turn to the main results of the paper, showing that if signaling occurs, then individual beliefs are decidedly non-Laplacian. In particular, Section 4.1 calculates the rank-order 
beliefs of an individual with arbitrary signal $x_{i}$ and shows that these beliefs are not uniform. Section 4.2 shows how this translates into the beliefs of the marginal individual with signal $x_{i}=x^{*}$ concerning the distribution of the aggregate action $A(y(\theta))$ and that these are likewise not uniform. Section 4.3 shows that in the limit as the signal precision $\alpha \rightarrow+\infty$, in which case there is strategic uncertainty but no fundamental uncertainty, the marginal individual believes the aggregate action $A(y(\theta))$ has a Bernoulli distribution on the extreme outcomes $\{0,1\}$, not a uniform distribution on the interval $[0,1]$.

\subsection{Rank-order beliefs with noisy signaling}

Consider the model with noisy signaling. Let $x^{*}, \theta^{*}$ denote the equilibrium thresholds and let $y(\theta)$ denote the equilibrium signaling policy.

Preliminaries. Conditional on $y(\theta)$ signals are IID normal with mean $y(\theta)$ and precision $\alpha$, so the proportion of individuals with signals less than $x_{i}$ is

$$
l\left(x_{i}, \theta\right)=\operatorname{Prob}\left[x_{j} \leq x_{i} \mid \theta\right]=\int_{-\infty}^{x_{i}} \sqrt{\alpha} \phi\left(\sqrt{\alpha}\left(x_{j}-y(\theta)\right) d x_{j}=\Phi\left(\sqrt{\alpha}\left(x_{i}-y(\theta)\right)\right)\right.
$$

Now let $G\left(k \mid x_{i}\right)$ denote the rank-order beliefs of an individual with signal $x_{i}$ in this signaling equilibrium. That is,

$$
G\left(k \mid x_{i}\right):=\operatorname{Prob}\left[l\left(x_{i}, \theta\right) \leq k \mid x_{i}, y(\cdot)\right]
$$

If there is noisy signaling in equilibrium (i.e., if $\alpha>\underline{\alpha}$ ), then the shape of the CDF $G\left(k \mid x_{i}\right)$ is crucially influenced by two key properties of the signaling policy $y(\theta)$, specifically (i) the discontinuity in $y(\theta)$ at the critical $\theta^{*}$, and (ii) the constancy $y(\theta)=y^{*}$ on the interval $\left(\theta^{*}, \theta^{* *}\right)$. To describe these properties of $G\left(k \mid x_{i}\right)$ precisely requires some notation for the critical proportions associated with the critical points $\theta^{*}, \theta^{* *}$ as viewed by an individual with signal $x_{i}$. In particular, let

$$
\bar{k}\left(x_{i}\right):=\lim _{\theta \uparrow \theta^{*}} l\left(x_{i}, \theta\right)=\Phi\left(\sqrt{\alpha}\left(x_{i}-\theta^{*}\right)\right)
$$

and

$$
\underline{k}\left(x_{i}\right):=\lim _{\theta \downarrow \theta^{* *}} l\left(x_{i}, \theta\right)=\Phi\left(\sqrt{\alpha}\left(x_{i}-\theta^{* *}\right)\right)
$$

The value $\bar{k}\left(x_{i}\right)$ is the equilibrium proportion of individuals with signals below $x_{i}$ in the limit as the true state $\theta$ approaches $\theta^{*}$ from below and likewise the value $\underline{k}\left(x_{i}\right)$ is the equilibrium proportion of individuals with signals below $x_{i}$ in the limit as the true state $\theta$ approaches $\theta^{* *}$ from above. Since the policy $y(\theta)$ is discontinuous at $\theta^{*}$, the former means evaluating $l\left(x_{i}, \theta\right)=\Phi\left(\sqrt{\alpha}\left(x_{i}-y(\theta)\right)\right)$ at $\lim _{\theta \uparrow \theta^{*}} y(\theta)=\theta^{*}$, not $y\left(\theta^{*}\right)=y^{*}>\theta^{*}$. By contrast, since $y(\theta)$ is continuous at $\theta^{* *}$, the latter means evaluating at $\lim _{\theta \downarrow \theta^{* *}} y(\theta)=\theta^{* *}$. Since $\theta^{*}<\theta^{* *}$ these proportions satisfy $\bar{k}\left(x_{i}\right)>\underline{k}\left(x_{i}\right)$ for all $x_{i}$, with both proportions strictly increasing in $x_{i}$. 
Now re-write the posterior density (??) for an individual with signal $x_{i}$ as

$$
\pi\left(\theta \mid x_{i}\right)=\frac{\sqrt{\alpha} \phi\left(\sqrt{\alpha}\left(x_{i}-y(\theta)\right)\right)}{m\left(x_{i}\right)}
$$

where $m\left(x_{i}\right)$ is the marginal factor that ensures $\pi\left(\theta \mid x_{i}\right)$ integrates to one, specifically

$$
m\left(x_{i}\right):=\int_{-\infty}^{\infty} \sqrt{\alpha} \phi\left(\sqrt{\alpha}\left(x_{i}-y(\theta)\right)\right) d \theta
$$

Equilibrium rank-order beliefs. With this notation in hand, and as detailed in the Appendix, it is straightforward to calculate the rank-order CDF. In particular:

Proposition 1. The rank-order beliefs of an individual with signal $x_{i}$ are given by

$$
G\left(k \mid x_{i}\right)= \begin{cases}\frac{1}{m\left(x_{i}\right)} k & k \in\left[0, \underline{k}\left(x_{i}\right)\right) \\ \frac{1}{m\left(x_{i}\right)} \underline{k}\left(x_{i}\right)+J\left(x_{i}\right) & k \in\left[\underline{k}\left(x_{i}\right), \bar{k}\left(x_{i}\right)\right) \\ \frac{1}{m\left(x_{i}\right)}\left[k+\underline{k}\left(x_{i}\right)-\bar{k}\left(x_{i}\right)\right]+J\left(x_{i}\right) & k \in\left[\bar{k}\left(x_{i}\right), 1\right]\end{cases}
$$

where

$$
J\left(x_{i}\right):=\frac{\sqrt{\alpha} \phi\left(\sqrt{\alpha}\left(x_{i}-\theta^{* *}\right)\right)}{m\left(x_{i}\right)}\left(\theta^{* *}-\theta^{*}\right)
$$

Figure 2 illustrates. At first, for $k \in\left[0, \underline{k}\left(x_{i}\right)\right)$, the CDF is linearly increasing in $k$ with slope $1 / m\left(x_{i}\right)$. At the critical proportion $k=\underline{k}\left(x_{i}\right)$ the probability jumps up discretely by the amount $J\left(x_{i}\right)$. It is then constant on the interval $k \in\left(\underline{k}\left(x_{i}\right), \bar{k}\left(x_{i}\right)\right)$ before again linearly increasing with slope $1 / m\left(x_{i}\right)$ for the remaining $k \in\left[\bar{k}\left(x_{i}\right), 1\right]$. Manipulating the definitions of $\underline{k}\left(x_{i}\right), \bar{k}\left(x_{i}\right)$ and $J\left(x_{i}\right)$ it is straightforward to see that $G\left(k \mid x_{i}\right)$ is continuous at $k=\bar{k}\left(x_{i}\right)$.

The jump at $k=\underline{k}\left(x_{i}\right)$ is inherited from the discrete change in the signaling policy from $y(\theta)=\theta$ to $y(\theta)=y^{*}=\theta^{* *}>\theta^{*}$ at the critical threshold $\theta^{*}$. This puts an atom, a discrete amount of probability mass, at the point $k=\underline{k}\left(x_{i}\right)$. The size of the jump

$$
J\left(x_{i}\right):=\frac{\sqrt{\alpha} \phi\left(\sqrt{\alpha}\left(x_{i}-\theta^{* *}\right)\right)}{m\left(x_{i}\right)}\left(\theta^{* *}-\theta^{*}\right)=\pi\left(\theta^{* *} \mid x_{i}\right)\left(\theta^{* *}-\theta^{*}\right)
$$

is the product of two terms, (i) the size of the bias $y\left(\theta^{*}\right)-\theta^{*}=\theta^{* *}-\theta^{*}$ achieved by the marginal policy-maker, a term that is the same for all individuals, and (ii) the posterior likelihood of $\theta^{* *}$, a term that depends on the individual's idiosyncratic signal realization. Following this jump, the CDF is constant on the open interval $\left(\underline{k}\left(x_{i}\right), \bar{k}\left(x_{i}\right)\right)$. This implies that the support of the rank-order distribution is not connected, the interval $\left(\underline{k}\left(x_{i}\right), \bar{k}\left(x_{i}\right)\right)$ has zero probability. This gap in the support of the rank-order beliefs is inherited from the constancy of the signaling policy on $\left(\theta^{*}, \theta^{* *}\right)$. In short, probability mass that would be 


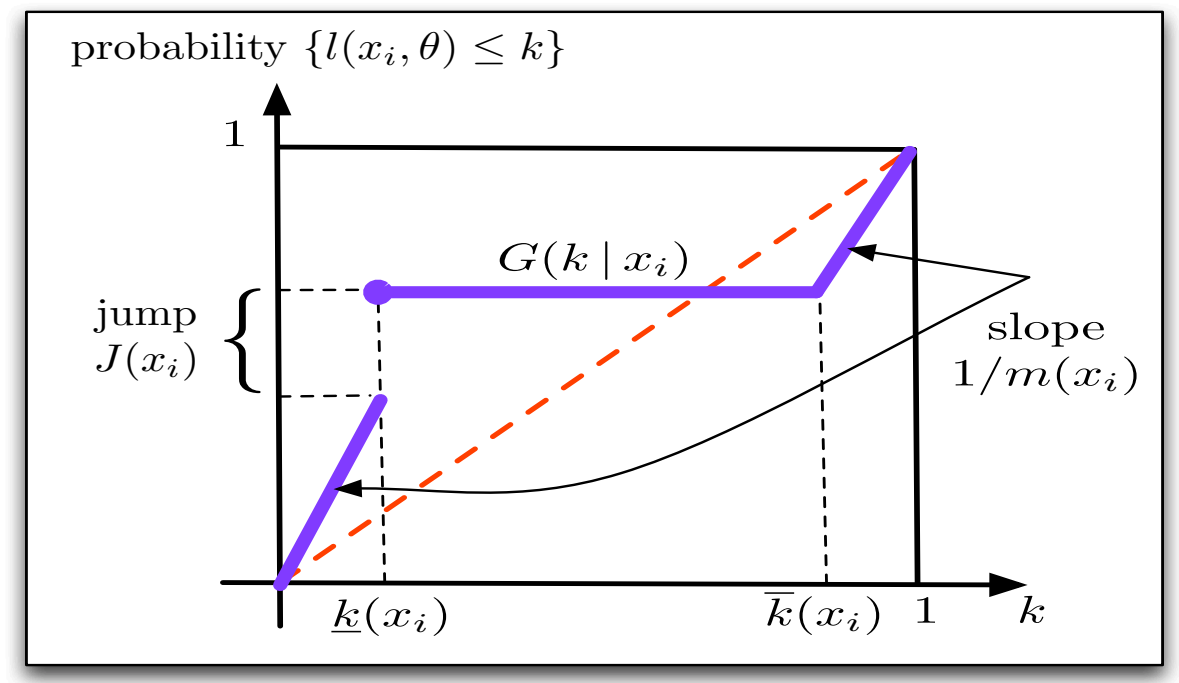

Figure 2: Rank beliefs of individual with signal $x_{i}$.

Probability $G\left(k \mid x_{i}\right)$ assigned to the proportion $l\left(x_{i}, \theta\right)$ of individuals with signals less than $x_{i}$ being less than $k$, i.e., beliefs about an individual's rank-order in the population. This is linearly increasing with slope $1 / m\left(x_{i}\right)$ for $k \in\left[0, \underline{k}\left(x_{i}\right)\right)$, jumps discretely by the amount $J\left(x_{i}\right)$ at $k=\underline{k}\left(x_{i}\right)$ (the beliefs have an atom at that point), is then constant on the interval $\left(\underline{k}\left(x_{i}\right), \bar{k}\left(x_{i}\right)\right.$ ), before again linearly increasing with slope $1 / m\left(x_{i}\right)$. A standard global game has $G\left(k \mid x_{i}\right)=k$, as indicated by the $45^{\circ}$-line.

associated with the interval $\left(\theta^{*}, \theta^{* *}\right)$ in the absence of signaling is now concentrated on a single point associated with $\theta^{*}$.

In the special case of no signaling (i.e., $\alpha<\underline{\alpha}$ ) so that $y(\theta)=\theta$, this reduces to a standard global game. In particular, we then have $m\left(x_{i}\right)=1$ for all $x_{i}$ and $\underline{k}\left(x_{i}\right)=\bar{k}\left(x_{i}\right)$ so that the jump disappears, as does the flat region, leaving us with the benchmark Laplacian beliefs that are independent of $x_{i}$ and uniform in $k$ on $[0,1]$. The equilibrium beliefs with signaling violate both these key properties of the benchmark, in particular (i) the signal realization $x_{i}$ is informative about rank-order, and (ii) the beliefs are not uniform on their support, the density is constant at $1 / m\left(x_{i}\right)$ everywhere on the support except at the atom $k=\underline{k}\left(x_{i}\right)$.

In the absence of noisy signaling, individuals have uniform rank-order beliefs because they begin with uninformative priors and their signal realization, while anchoring their expectations about $\theta$, does not provide them with any information on the proportion of individuals who observe higher (or lower) signals. With noisy signaling, however, the policy-maker's preferred direction for bias plus common knowledge of the costly signaling technology and the circumstances under which it will be used in equilibrium combine to make an individual's signal partially informative about their rank-order.

Cross-section of marginals. In general the slope $1 / m\left(x_{i}\right)$ of the rank-order beliefs varies smoothly with the signal realization and may be greater or less than one. The marginal factor $m\left(x_{i}\right)$ is a measure of the change in the likelihood of the event $\left\{\theta<\theta^{*}\right\}$ between an equilibrium with and without signaling. In particular, we can use (20) to rewrite the 
individual beliefs (8) as

$$
m\left(x_{i}\right)=\frac{\operatorname{Prob}\left[\theta<\theta^{*} \mid x_{i}, b(\cdot)=0\right]}{\operatorname{Prob}\left[\theta<\theta^{*} \mid x_{i}, y(\cdot)\right]}
$$

that is, the ratio of the belief that $\left\{\theta<\theta^{*}\right\}$ under the (generally incorrect) presumption that there is no bias, $b(\theta)=0$, to the belief that $\left\{\theta<\theta^{*}\right\}$ under the correct equilibrium beliefs internalizing the signaling policy $y(\theta)$. An individual with $m\left(x_{i}\right)<1$ assigns more belief to the status quo being overturned once they internalize the signaling policy while an individual with $m\left(x_{i}\right)>1$ assigns less belief to the status quo being overturned once they internalize the signaling policy.

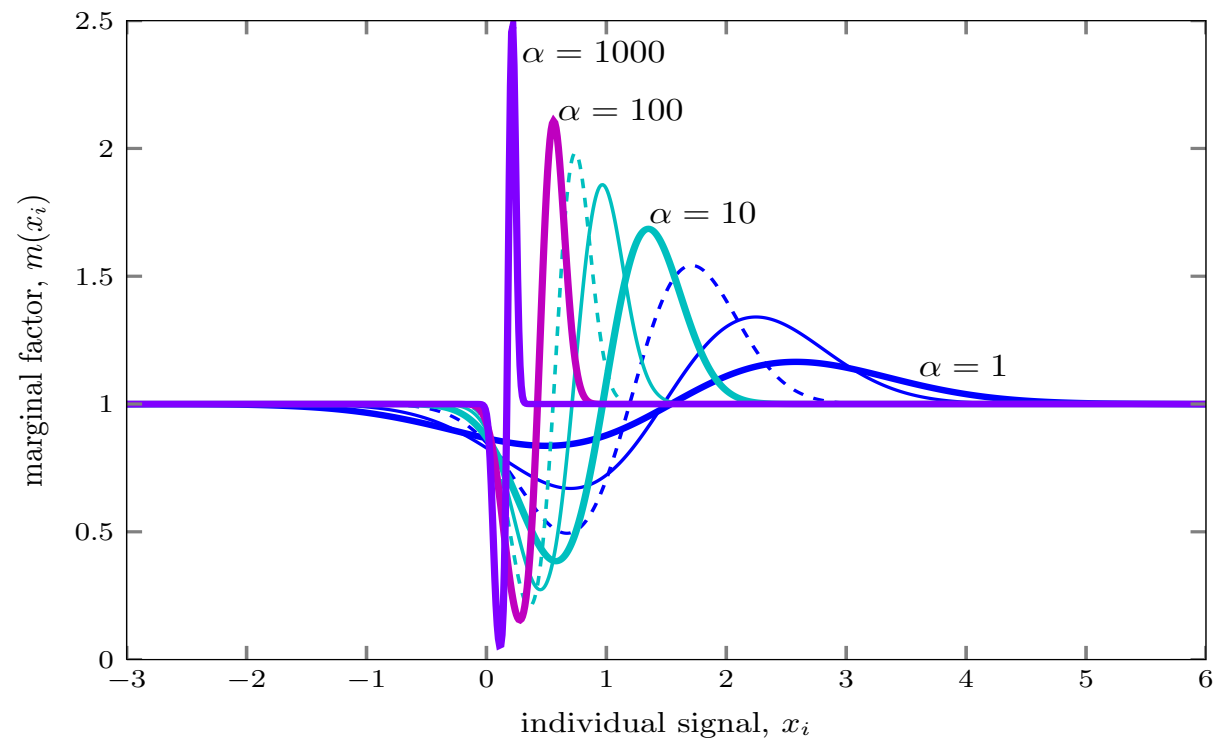

Figure 3: Marginal factor $m\left(x_{i}\right)$ for individual with signal $x_{i}$.

The marginal factors are a measure of the change in likelihood of $\left\{\theta<\theta^{*}\right\}$ induced by the signaling. When $m\left(x_{i}\right)<1$, as is the case for low $x_{i}$, an individual assigns less belief to $\left\{\theta<\theta^{*}\right\}$ once they internalize the signaling policy. When $m\left(x_{i}\right)>1$, as is the case for high $x_{i}$, an individual assigns more belief to $\left\{\theta<\theta^{*}\right\}$ once they internalize the signaling policy. As the signal precision $\alpha$ increases, the marginals in the critical region become increasingly polarized. That is, an individual with low $m\left(x_{i}\right)$ has rank-order beliefs that give relatively high likelihood to the extreme points $k=0$ and $k=1$. By contrast, an individual with a high $m\left(x_{i}\right)$ tends to give relatively low likelihood to these extremes.

The general shape of the marginal factor $m\left(x_{i}\right)$ is shown in Figure 3. To understand this shape, observe that when there is no possibility of signaling, $m\left(x_{i}\right)$ is always one. Even with signaling, extreme realizations of $x_{i}$ continue to imply $m\left(x_{i}\right)$ close to one. An individual with an extreme realization has essentially the same beliefs about $\left\{\theta<\theta^{*}\right\}$ irrespective of the possibility of signaling: a sufficiently low $x_{i}$ will make them quite sure $\theta$ is low and that the status quo will be overturned, likewise a sufficiently high $x_{i}$ will make them quite sure $\theta$ is high and that the status quo will prevail. The marginal $m\left(x_{i}\right)$ is less than one for low $x_{i}$. This is because the policy-maker's incentives are to bias the signals to the high side, so a low realized $x_{i}$ may reflect a low true $\theta$ without bias or an even lower $\theta$ with bias - so an individual with low $x_{i}$ assigns more weight to low values of $\theta$ and hence gives more belief to the status quo being overturned. Similarly the marginal is greater than one for high $x_{i}$. This 
reflects an increasing belief that $\theta$ is not low. In particular, a high realized $x_{i}$ may reflect a high true $\theta$ or a moderate $\theta$ with bias - so an individual with high $x_{i}$ assigns more weight to high values of $\theta$ and hence gives less belief to the status quo being overturned.

Increasingly polarized beliefs. Figure 3 also shows how $m\left(x_{i}\right)$ changes as the signal precision $\alpha$ increases. As $\alpha$ increases, the zone where $m\left(x_{i}\right)$ differs appreciably from one shrinks, but inside that critical zone the marginal factors become more polarized. In particular, an individual with low $m\left(x_{i}\right)$ has high slope $1 / m\left(x_{i}\right)$ and hence has rank-order beliefs $G\left(k \mid x_{i}\right)$ that give relatively high likelihood to proportions $k$ near the extreme points $k=0$ and $k=1$. By contrast, an individual with a very high $m\left(x_{i}\right)$ tends to give relatively low likelihood to proportions near the extremes. This tension is present at all levels of $\alpha$ (such that $\alpha>\underline{\alpha}$ ), but is amplified in the extreme as $\alpha \rightarrow+\infty$.

\subsection{Beliefs about the aggregate action with noisy signaling}

As in a standard global game, the rank-order beliefs translate quite directly into the beliefs of the marginal individual about the aggregate action $A(y(\theta))$. In particular, the marginal individual has signal $x_{i}=x^{*}$ and believes the aggregate action $A(y(\theta))=l\left(x^{*}, \theta\right)$ is distributed according to the $\mathrm{CDF}$

$$
G^{*}(k):=G\left(k \mid x^{*}\right)=\operatorname{Prob}\left[A(y(\theta)) \leq k \mid x^{*}\right]
$$

And for the individual with $x_{i}=x^{*}$ we can calculate that

$$
m\left(x^{*}\right)=\int_{-\infty}^{\infty} \sqrt{\alpha} \phi\left(\sqrt{\alpha}\left(x^{*}-y(\theta)\right)\right) d \theta=\frac{\theta^{*}}{1-p}
$$

where the last equality follows from rearranging the indifference conditions (7)-(8) and using the definition of $m\left(x_{i}\right)$ from (20). Thus the marginal individual has $m\left(x^{*}\right)<1$ if and only if signaling is effective, $\theta^{*}<1-p$. This correspondence between $m\left(x^{*}\right)$ and the ratio $\theta^{*} /(1-p)$ is natural. Recall from $(24)$ that $m\left(x^{*}\right)<1$ simply means that the marginal individual assigns more belief to $\left\{\theta<\theta^{*}\right\}$ once they internalize the equilibrium signaling policy $y(\theta)$. For the signaling to be effective for the policy-maker, it must be the case that the marginal individual is 'over confident' in this manner.

Now using this simple expression for $m\left(x^{*}\right)$ in the general formula (21) above gives: 
Proposition 2. The marginal individual with signal $x^{*}$ believes the aggregate action $A(y(\theta))$ has distribution

$$
G^{*}(k)= \begin{cases}\frac{1-p}{\theta^{*}} k & k \in\left[0, k^{* *}\right) \\ 1-p & k \in\left[k^{* *}, k^{*}\right) \\ \frac{1-p}{\theta^{*}}(k-1)+1 & k \in\left[k^{*}, 1\right]\end{cases}
$$

with critical proportions $k^{* *}:=\underline{k}\left(x^{*}\right)$ and $k^{*}:=\bar{k}\left(x^{*}\right)$.

Qualitatively, this CDF has the same shape as the general rank-order beliefs. It is first linearly increasing, then jumps (at the critical proportion $k^{* *}$ ), is then constant on $\left(k^{* *}, k^{*}\right)$, before again linearly increasing on the remaining $\left[k^{*}, 1\right]$. Using (7) and (8) above we also have that $k^{*}$ and the critical threshold $\theta^{*}$ are related by

$$
k^{*}:=\bar{k}\left(x^{*}\right)=\Phi\left(\sqrt{\alpha}\left(x^{*}-\theta^{*}\right)\right)=1-\frac{p}{1-p} \theta^{*}
$$

which can be used to verify that $G^{*}(k)$ is continuous at $k=k^{*}$, Similarly, $k^{* *}$ is given by

$$
k^{* *}:=\underline{k}\left(x^{*}\right)=\Phi\left(\sqrt{\alpha}\left(x^{*}-\theta^{* *}\right)\right)=\Phi(-\sqrt{\alpha} \gamma)=\Phi\left(-\sqrt{2 \log \left(\frac{\sqrt{\alpha}}{c} \phi(0)\right)}\right)
$$

which is the size of the aggregate action corresponding to any policy-maker that intervenes.

Two equilibrium configurations. There are two equilibrium configurations to consider. In both configurations, there is probability mass $1-p$ associated with the lower closed interval $\left[0, k^{* *}\right]$, including the atom at $k=k^{* *}$, and complementary mass $p$ associated with the upper closed interval $\left[k^{*}, 1\right]$. In short, independent of other parameters,

$$
\begin{aligned}
G^{*}\left(k^{* *}\right) & =\operatorname{Prob}\left[k \leq k^{* *} \mid x^{*}\right]=1-p \\
1-G^{*}\left(k^{*}\right) & =\operatorname{Prob}\left[k \geq k^{*} \mid x^{*}\right]=p
\end{aligned}
$$

while the open interval $\left(k^{* *}, k^{*}\right)$ in between has zero probability mass in both configurations.

As illustrated in Figure 4, these two configurations differ in the magnitudes of the critical points $k^{*}, k^{* *}$ and hence differ in the how the probability masses $\{1-p, p\}$ are allocated within each of these outer intervals. In the first configuration, signaling is effective, so that $\theta^{*}$ is less than the Morris-Shin benchmark $\theta_{\mathrm{MS}}^{*}=1-p$, the marginal individual has $m\left(x^{*}\right)=\theta^{*} /(1-p)<1$, and the density $1 / m\left(x^{*}\right)=(1-p) / \theta^{*}>1$ is relatively high, i.e., higher than the uniform density benchmark. In the second configuration, signaling is ineffective, so that $\theta^{*}$ is greater than the Morris-Shin benchmark $1-p$, the marginal individual has $m\left(x^{*}\right)=\theta^{*} /(1-p)>1$, and the density $1 / m\left(x^{*}\right)=(1-p) / \theta^{*}<1$ is relatively low, i.e., lower than the uniform density benchmark. Since the total probability mass allocated over 
the outer intervals is the same in both configurations, these differences in density are achieved by having shorter intervals $\left[0, k^{* *}\right]$ and $\left[k^{*}, 1\right]$ in the first case and longer intervals in the second so as to preserve the total masses at $1-p$ and $p$ respectively. Equivalently, the inner interval $\left(k^{* *}, k^{*}\right)$ that attracts zero probability mass is wider in the effective signaling configuration and narrower in the ineffective signaling configuration. In the absence of signaling altogether, the inner interval disappears to leave the CDF lying on the $45^{\circ}$-line.

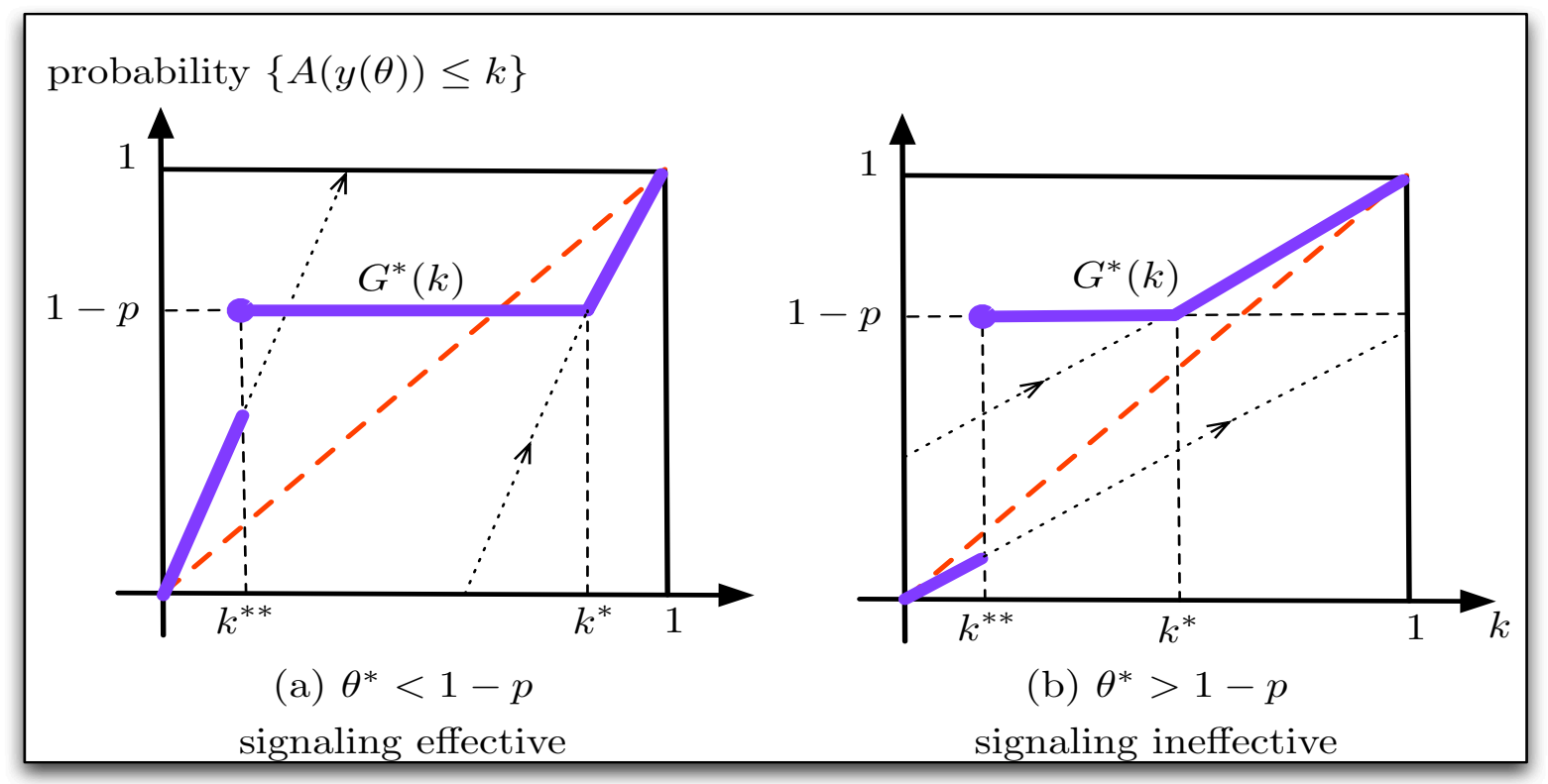

Figure 4: Beliefs about the aggregate action $A(y(\theta))$.

The shape of the beliefs $G^{*}(k):=\operatorname{Prob}\left[A(y(\theta)) \leq k \mid x^{*}\right]$ depends on whether the threshold $\theta^{*}$ is above or below the Morris-Shin benchmark $1-p$. If parameters are such that $\theta^{*}<1-p$, then signaling is effective and the CDF is as in panel (a). Otherwise, if $\theta^{*}>1-p$, signaling is ineffective and the CDF is as in panel (b). In both panels the lower closed interval [0, $\left.k^{* *}\right]$ has probability mass $1-p$ while the upper closed interval $\left[k^{*}, 1\right]$ has probability mass $p$ (and so the inner open interval $\left(k^{* *}, k^{*}\right.$ ) has zero probability mass). In panel (a) the outer intervals are short but the density $(1-p) / \theta^{*}$ is greater than one. In panel (b) the outer intervals are longer but the density is less than one. A standard global game has $G^{*}(k)=k$, as indicated by the $45^{\circ}$ line, with density equal to one for all $k \in[0,1]$.

\subsection{Bernoulli limit}

These non-uniform beliefs about $A(y(\theta))$ are even more striking when we consider the limit as the signal precision $\alpha \rightarrow+\infty$. In this limit there is no longer any fundamental uncertainty, all that remains is strategic uncertainty.

Limiting equilibrium beliefs. Recall from Lemma 1 above that in the limit as $\alpha \rightarrow+\infty$, the critical threshold $\theta^{*} \rightarrow 0^{+}$so that equilibrium outcomes break decisively in the policymaker's favor. This extremely effective signaling has crucial implications for equilibrium beliefs. In particular, observe that this means the marginal individual has $m\left(x^{*}\right)=\theta^{*} /(1-$ $p) \rightarrow 0^{+}$(as shown in Figure 3 above). Thus, this individual assigns very high density to 
outcomes in the lower and upper intervals $\left[0, k^{* *}\right]$ and $\left[k^{*}, 1\right]$. In fact, as the signal precision becomes sufficiently high these intervals collapse so that:

Proposition 3. In the limit as the signal precision $\alpha \rightarrow+\infty$ the beliefs of the marginal individual $G^{*}(k)$ approach the Bernoulli distribution on the extreme outcomes $\{0,1\}$ with probabilities $\{1-p, p\}$ respectively. That is,

$$
\begin{aligned}
& \operatorname{Prob}\left[A(y(\theta))=0 \mid x^{*}\right]=1-p \\
& \operatorname{Prob}\left[A(y(\theta))=1 \mid x^{*}\right]=p
\end{aligned}
$$

Figure 5 illustrates. In the limit as $\alpha \rightarrow+\infty$, the critical points $k^{* *} \rightarrow 0$ and $k^{*} \rightarrow 1$ so that the lower interval collapses to the extreme lower bound, $\left[0, k^{* *}\right] \rightarrow\{0\}$, while the upper interval likewise collapses to the extreme upper bound, $\left[k^{*}, 1\right] \rightarrow\{1\}$. Since the probability mass associated with the lower interval is $1-p$ while the probability mass associated with the upper interval is $p$, both independent of $\alpha$, in the limit we simply have the belief that $A(y(\theta))$ has the Bernoulli distribution on the extreme outcomes $\{0,1\}$ with probabilities $\{1-p, p\}$. This is about as different from uniform beliefs on $[0,1]$ as could possibly be imagined. Rather than giving all aggregate actions equal likelihood, the marginal individual gives weight only to the polar extreme outcomes $\{0,1\}$.

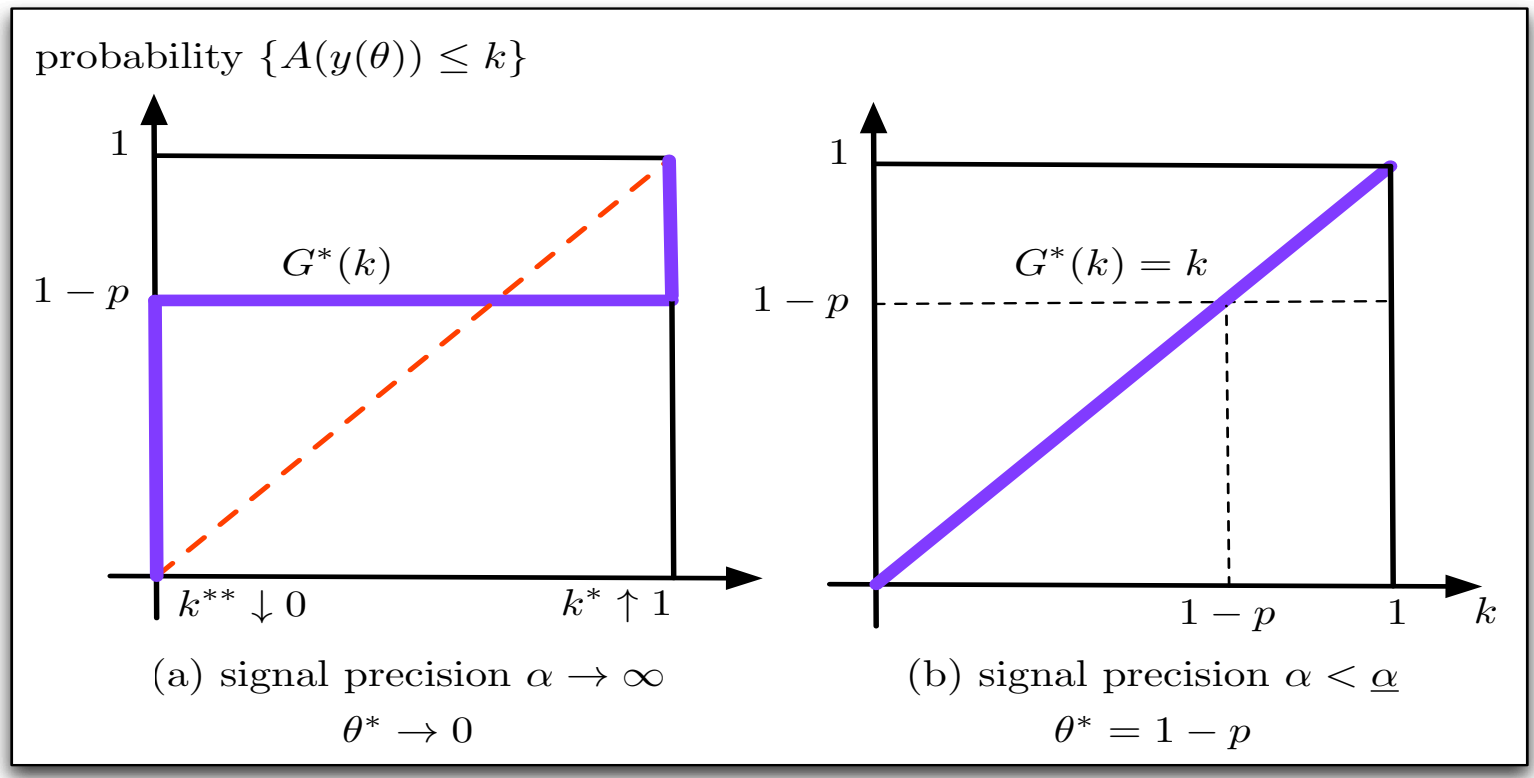

Figure 5: Bernoulli and uniform limiting cases.

The shape of the beliefs $G^{*}(k):=\operatorname{Prob}\left[A(y(\theta)) \leq k \mid x^{*}\right]$ for high and low signal precision. As the signal precision $\alpha \rightarrow \infty$, the threshold $\theta^{*} \rightarrow 0$. In this case $k^{* *} \rightarrow 0$ from above while $k^{*} \rightarrow 1$ from below so that the lower interval $\left[0, k^{* *}\right] \rightarrow\{0\}$ while the upper interval $\left[k^{*}, 1\right] \rightarrow\{1\}$. Since the probabilities attached to these intervals are $p$ and $1-p$ independent of $\alpha$, in the limit, the beliefs approach the Bernoulli distribution on the extreme outcomes $\{0,1\}$ with probabilities $\{1-p, p\}$ respectively. For low signal precision, $\alpha \leq \underline{\alpha}$, there is no signaling in equilibrium. In this case, $k^{* *}=k^{*}=1-p$ and $G^{*}(k)$ is uniform on [0,1]. 
The intuition for this stark result is that, for high enough $\alpha$, the marginal individual has a signal $x^{*}$ that is in the gap $\left(\theta^{*}, \theta^{* *}\right)$ - i.e., not in the support of the equilibrium beliefs over $A(y(\theta))$. Given this, the marginal individual assigns non-vanishing probability mass only to the two values of $y(\theta)$ at the edges of the gap, i.e., to the extremes $y\left(\theta^{*}\right)=\theta^{* *}$ and $y\left(\theta^{* *}\right)=\theta^{*}$ (as per Figure 1) and hence to the two extreme values of the aggregate action $A(y(\theta))$. Put differently, in this model equilibrium beliefs always have one atom, a discrete amount of probability mass, corresponding to $\theta^{*}$, that is inherited from the discontinuity in the signal policy $y(\theta)$. Proposition 3 then says that in the limit as $\alpha \rightarrow \infty$ there is a second atom corresponding to $\theta^{* *}$ and that together these two atoms are exhaustive.

Examples. In a standard global game the expected aggregate action is $\mathbb{E}\left[A(\theta) \mid x^{*}\right]=$ $1 / 2$, independent of parameters, ${ }^{11}$ but here $\mathbb{E}\left[A(y(\theta)) \mid x^{*}\right]=p$. Notice that the expected aggregate action is increasing in the individual opportunity cost $p$, so even though a higher $p$ makes any individual less likely to take the risky action, conditional on being marginal, a higher $p$ increases belief that $A(y(\theta))$ is high. Similarly, whereas in a standard global game the variance of the aggregate action is $\operatorname{Var}\left[A(\theta) \mid x^{*}\right]=1 / 12$, here the variance is $\operatorname{Var}\left[A(y(\theta)) \mid x^{*}\right]=p(1-p)$ which varies from 0 to $1 / 4$ depending on how skewed the Bernoulli is. In the symmetric case where $p=1 / 2$, so that the uniform and Bernoulli beliefs have the same mean, the variance for the Bernoulli is larger than that of the uniform (1/4 vs. 1/12). More generally, so long as $p$ is not too extreme the marginal individual is more uncertain about the aggregate outcome $A(y(\theta))$ than they would be absent the noisy signaling.

In short, the endogenous information structure in the global game with noisy signaling leads to equilibrium beliefs that no-longer have the Laplacian properties familiar from standard global games. The departure from Laplacian beliefs manifests itself in three interrelated ways: (i) individuals are not agnostic about their rank-order in the population, consequently (ii) in particular, the marginal individual who is indifferent about taking the risky action does not believe the aggregate action is uniformly distributed, and (iii) in the limit as fundamental uncertainty vanishes, the marginal individual has Bernoulli beliefs, rather than giving all aggregate actions equal likelihood, they give weight only to the extreme outcomes $\{0,1\}$.

Subtle probabilistic reasoning after all. What is the upshot of these non-Laplacian beliefs? Recall that in standard global games individuals do not need to use subtle probabilistic reasoning to be taking actions that are optimal - in standard global games an individual who chooses an action $a_{i}$ that is a best response to the belief that the aggregate action $A$ is uniformly distributed will in fact be behaving optimally (Morris and Shin, 2003). In this

\footnotetext{
${ }^{11}$ That is, model parameters determine the identity of the marginal $x^{*}$ but do not influence the size of the expected aggregate action conditional on $x^{*}$.
} 
sense, the Laplacian scenario opens the door to the possibility of sustaining a relatively 'robust' equilibrium that does not require a perhaps implausible degree of rationality on the part of the information receivers. By contrast, in this game with noisy signaling, optimal play most certainly does require agents to use subtle probabilistic reasoning (that internalizes the entire equilibrium fixed-point problem).

\section{Conclusion}

This paper provides a complete analytic characterization of the equilibrium beliefs of individuals playing a binary action global game with noisy signaling. The underlying standard global game features Laplacian beliefs, beliefs that are essentially agnostic as to an individual's position in the population and hence similarly agnostic as to the size of the aggregate action. By contrast, the global game with noisy signaling features beliefs that are strikingly non-Laplacian. In the standard global game, an individual will be behaving optimally if they take the action that constitutes a best response to the naive belief that the aggregate action is uniformly distributed. By contrast, in the game with noisy signaling, such Laplacian actions will generally be distinctly sub-optimal. Since the equilibrium with noisy signaling is otherwise very similar to that obtained in a standard global game, this example suggests that the Laplacian property is less an intrinsic property of this class of games and rather more a consequence of the fairly simple information structures that are generally used in global games applications. 


\section{Appendix}

\section{Proof of Lemma 1.}

This is the last part of Proposition 3 in Edmond (2013) and is proved in Appendix A.4 to that paper.

\section{Proof of Proposition 1.}

Conditional on $y(\theta)$ the proportion of individuals with signals less than $x_{i}$ is $l\left(x_{i}, \theta\right):=\operatorname{Prob}\left[x_{j} \leq x_{i} \mid \theta\right]=$ $\Phi\left(\sqrt{\alpha}\left(x_{i}-y(\theta)\right)\right)$ where $y(\theta)=y^{*}$ for $\theta \in\left[\theta^{*}, \theta^{* *}\right)$ and $y(\theta)=\theta$ otherwise. Using the expression (??) for the posterior density of an individual with signal $x_{i} \in \mathbb{R}$, the rank-order CDF can be calculated from

$$
G\left(k \mid x_{i}\right):=\operatorname{Prob}\left[l\left(x_{i}, \theta\right) \leq k \mid y(\cdot)\right]=\int_{\Theta\left(k, x_{i}\right)} \frac{\sqrt{\alpha} \phi\left(\sqrt{\alpha}\left(x_{i}-y(\theta)\right)\right.}{m\left(x_{i}\right)} d \theta
$$

where

$$
\Theta\left(k, x_{i}\right):=\left\{\theta \in \mathbb{R} \mid l\left(x_{i}, \theta\right) \leq k\right\}, \quad k \in[0,1]
$$

and where $m\left(x_{i}\right)$ is the marginal factor, as defined in (20) above. To calculate this integral we make use of the piecewise nature of the signaling policy $y(\theta)$. To do this, recall the critical points $\underline{k}\left(x_{i}\right), \bar{k}\left(x_{i}\right)$ as defined in (17)-(18) above and partition the interval $[0,1]$ into the following three disjoint subsets: (i) the interval $\left[0, \underline{k}\left(x_{i}\right)\right)$, (ii) the interval $\left[\underline{k}\left(x_{i}\right), \bar{k}\left(x_{i}\right)\right)$ and (iii) the interval $\left[\bar{k}\left(x_{i}\right), 1\right]$. Inspecting Figure 6 we see that if, for example, we take some $k$ in the first interval $\left[0, \underline{k}\left(x_{i}\right)\right)$ on the vertical axis then that corresponds to some policy-maker type $\theta \geq \theta^{* *}$ for which $y(\theta)=\theta$. Most importantly, if we take the singular point $\underline{k}\left(x_{i}\right)$ then that corresponds to the entire interval $\left[\theta^{*}, \theta^{* *}\right)$. We calculate the CDF $G\left(k \mid x_{i}\right)$ considering in turn values of $k$ corresponding to each of the three cases:

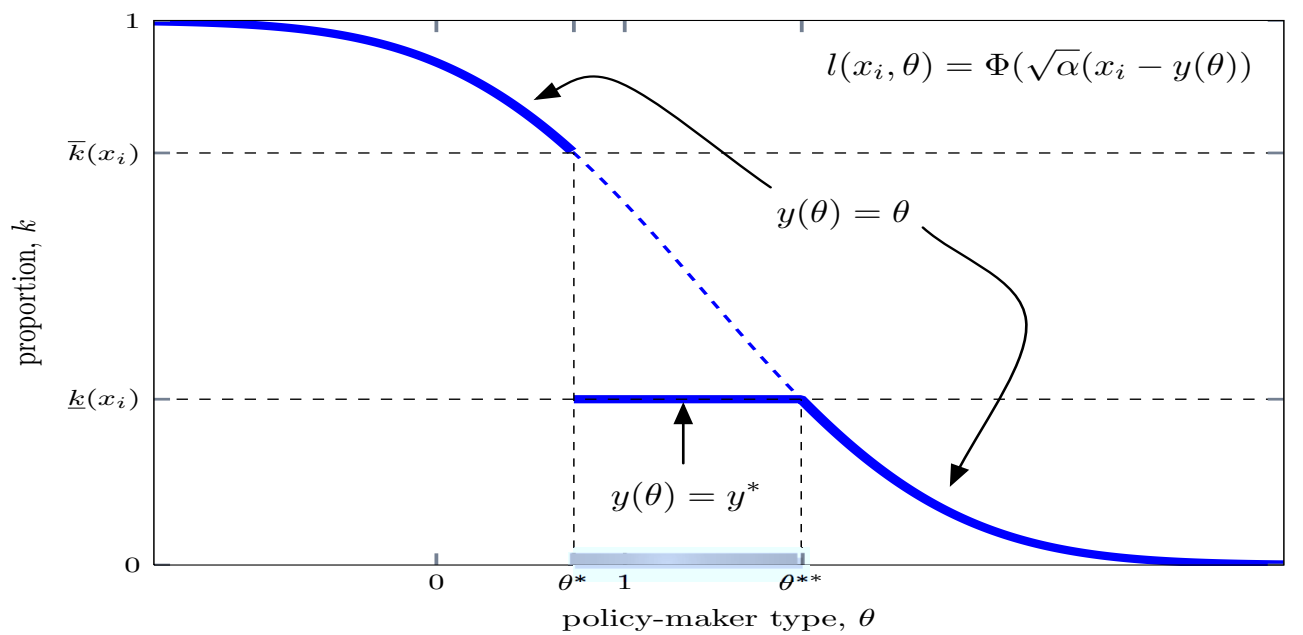

Figure 6: Constructing the rank-order beliefs $G\left(k \mid x_{i}\right)$.

The proportion of individuals with signals less than $x_{i}$ is $l\left(x_{i}, \theta\right):=\operatorname{Prob}\left[x_{j} \leq x_{i} \mid \theta\right]=\Phi\left(\sqrt{\alpha}\left(x_{i}-y(\theta)\right)\right)$ where $y(\theta)=y^{*}$ for $\theta \in\left[\theta^{*}, \theta^{* *}\right)$ and $y(\theta)=\theta$ otherwise. The interval $[0,1]$ on the vertical axis is partitioned into three disjoint subsets demarcated by the critical points $\underline{k}\left(x_{i}\right), \bar{k}\left(x_{i}\right)$ and the CDF $G\left(k \mid x_{i}\right)$ is calculated by considering in turn values of $k$ corresponding to each of these three subsets. Of most importance is the singular point $\underline{k}\left(x_{i}\right)$ that corresponds to the entire interval $\left[\theta^{*}, \theta^{* *}\right)$.

(i) Observe from Figure 6 that for any $k \in\left[0, \underline{k}\left(x_{i}\right)\right)$ we are considering only policy-maker types for which $y(\theta)=\theta$. Let $\hat{\theta}\left(k, x_{i}\right):=x_{i}-\Phi^{-1}(k) / \sqrt{\alpha}$ so that $l\left(x_{i}, \theta\right) \leq k$ if and only if $\theta \geq \hat{\theta}\left(k, x_{i}\right)$. Hence on this 
interval of $k$ the CDF takes the values

$$
\text { for } k \in\left[0, \underline{k}\left(x_{i}\right)\right) \quad \begin{aligned}
G\left(k \mid x_{i}\right) & =\int_{\hat{\theta}\left(k, x_{i}\right)}^{\infty} \frac{\sqrt{\alpha} \phi\left(\sqrt{\alpha}\left(x_{i}-\theta\right)\right.}{m\left(x_{i}\right)} d \theta \\
& =\frac{1}{m\left(x_{i}\right)}\left(1-\Phi\left(\sqrt{\alpha}\left(x_{i}-\frac{1}{\sqrt{\alpha}} \Phi^{-1}(k)-x_{i}\right)\right)\right) \\
& =\frac{1}{m\left(x_{i}\right)} k
\end{aligned}
$$

(ii) For $k \in\left[\underline{k}\left(x_{i}\right), \bar{k}\left(x_{i}\right)\right)$ we begin with the probability mass $G\left(\underline{k}\left(x_{i}\right) \mid x_{i}\right)$ corresponding to the end of the first subset above in case (i) and then add to that mass at the singular point $\underline{k}\left(x_{i}\right)$ corresponding to the interval $\left[\theta^{*}, \theta^{* *}\right)$. The remaining proportions, namely the open interval $\left(\underline{k}\left(x_{i}\right), \bar{k}\left(x_{i}\right)\right)$, are not in the support of the rank-order beliefs. Thus the CDF is constant on this interval, always taking the value

$$
\text { for } k \in\left[\underline{k}\left(x_{i}\right), \bar{k}\left(x_{i}\right)\right) \quad \begin{aligned}
G\left(k \mid x_{i}\right) & =\frac{1}{m\left(x_{i}\right)} \underline{k}\left(x_{i}\right)+\int_{\theta^{*}}^{\theta^{* *}} \frac{\sqrt{\alpha} \phi\left(\sqrt{\alpha}\left(x_{i}-y^{*}\right)\right)}{m\left(x_{i}\right)} d \theta \\
& =\frac{1}{m\left(x_{i}\right)} \underline{k}\left(x_{i}\right)+J\left(x_{i}\right)
\end{aligned}
$$

where the jump term $J\left(x_{i}\right)$ is

$$
J\left(x_{i}\right)=\int_{\theta^{*}}^{\theta^{* *}} \frac{\sqrt{\alpha} \phi\left(\sqrt{\alpha}\left(x_{i}-y^{*}\right)\right)}{m\left(x_{i}\right)} d \theta=\frac{\sqrt{\alpha} \phi\left(\sqrt{\alpha}\left(x_{i}-\theta^{* *}\right)\right)}{m\left(x_{i}\right)}\left(\theta^{* *}-\theta^{*}\right)
$$

and where the last equality uses $y^{*}=\theta^{* *}$.

(iii) For $k \in\left[\bar{k}\left(x_{i}\right), 1\right]$ we add further probability mass corresponding to policy-maker types in the interval $\left(-\infty, \theta^{*}\right)$. For these types we are back to $y(\theta)=\theta$. Again let $\hat{\theta}\left(k, x_{i}\right):=x_{i}-\Phi^{-1}(k) / \sqrt{\alpha}$. Hence on this interval of $k$ the $\mathrm{CDF}$ takes the values

$$
\text { for } k \in\left[\bar{k}\left(x_{i}\right), 1\right] \quad \begin{aligned}
G\left(k \mid x_{i}\right) & =\frac{1}{m\left(x_{i}\right)} \underline{k}\left(x_{i}\right)+J\left(x_{i}\right)+\int_{\hat{\theta}\left(k, x_{i}\right)}^{\theta^{*}} \frac{\sqrt{\alpha} \phi\left(\sqrt{\alpha}\left(x_{i}-\theta\right)\right.}{m\left(x_{i}\right)} d \theta \\
& =\frac{1}{m\left(x_{i}\right)} \underline{k}\left(x_{i}\right)+J\left(x_{i}\right)+\frac{1}{m\left(x_{i}\right)}\left[\Phi\left(\sqrt{\alpha}\left(\theta^{*}-x_{i}\right)-\Phi\left(-\Phi^{-1}(k)\right)\right]\right. \\
& =\frac{1}{m\left(x_{i}\right)} \underline{k}\left(x_{i}\right)+J\left(x_{i}\right)+\frac{1}{m\left(x_{i}\right)}\left[\left(1-\bar{k}\left(x_{i}\right)\right)-(1-k)\right] \\
& =\frac{1}{m\left(x_{i}\right)}\left[k+\underline{k}\left(x_{i}\right)-\bar{k}\left(x_{i}\right)\right]+J\left(x_{i}\right)
\end{aligned}
$$

where the third equality uses $\underline{k}\left(x_{i}\right)=\Phi\left(\sqrt{\alpha}\left(x_{i}-\theta^{*}\right)\right.$, as in (17) above.

Together, equations (34)-(35)-(36) give the stated piecewise formula for the rank-order CDF $G\left(k \mid x_{i}\right)$.

\section{Miscellaneous equilibrium relationships}

The following relationships are used repeatedly in the proof of Proposition 2 below. The indifference condition of the marginal individual with signal $x_{i}=x^{*}$, equation (8), can be written

$$
\operatorname{Prob}\left[\theta<\theta^{*} \mid x^{*}, y(\cdot)\right]=\int_{-\infty}^{\theta^{*}} \frac{\sqrt{\alpha} \phi\left(x^{*}-\theta\right)}{m\left(x^{*}\right)} d \theta=\frac{\Phi\left(\sqrt{\alpha}\left(\theta^{*}-x^{*}\right)\right)}{m\left(x^{*}\right)}=p
$$

where

$$
\begin{aligned}
m\left(x^{*}\right) & =\int_{-\infty}^{\theta^{*}} \sqrt{\alpha} \phi\left(\sqrt{\alpha}\left(x^{*}-\theta\right)\right) d \theta+\int_{\theta^{*}}^{\infty} \sqrt{\alpha} \phi\left(\sqrt{\alpha}\left(x^{*}-y(\theta)\right)\right) d \theta \\
& =\Phi\left(\sqrt{\alpha}\left(\theta^{*}-x^{*}\right)\right)+\int_{\theta^{*}}^{\infty} \sqrt{\alpha} \phi\left(\sqrt{\alpha}\left(x^{*}-y(\theta)\right)\right) d \theta
\end{aligned}
$$


which, as usual, uses $y(\theta)=\theta$ for $\theta<\theta^{*}$. Hence the individual indifference condition (37) can be rearranged to obtain

$$
\begin{aligned}
\Phi\left(\sqrt{\alpha}\left(\theta^{*}-x^{*}\right)\right) & =\frac{p}{1-p} \int_{\theta^{*}}^{\infty} \sqrt{\alpha} \phi\left(\sqrt{\alpha}\left(x^{*}-y(\theta)\right)\right) d \theta \\
& =\frac{p}{1-p}\left[\int_{\theta^{*}}^{\theta^{* *}} \sqrt{\alpha} \phi\left(\sqrt{\alpha}\left(x^{*}-y^{*}\right)\right) d \theta+\int_{\theta^{* *}}^{\infty} \sqrt{\alpha} \phi\left(\sqrt{\alpha}\left(x^{*}-\theta\right)\right) d \theta\right] \\
& =\frac{p}{1-p}\left[\sqrt{\alpha} \phi\left(\sqrt{\alpha}\left(x^{*}-y^{*}\right)\right)\left(\theta^{* *}-\theta^{*}\right)+1-\Phi\left(\sqrt{\alpha}\left(\theta^{* *}-x^{*}\right)\right]\right.
\end{aligned}
$$

Now substituting in the properties of the signaling policy $y^{*}=\theta^{* *}=x^{*}+\gamma$, where the coefficient $\gamma$ solves $\sqrt{\alpha} \phi(\sqrt{\alpha} \gamma)=c$, we obtain the relationship

$$
\Phi\left(\sqrt{\alpha}\left(\theta^{*}-x^{*}\right)\right)=\frac{p}{1-p}\left[c\left(x^{*}+\gamma-\theta^{*}\right)+\Phi(-\sqrt{\alpha} \gamma)\right]
$$

The indifference condition of the marginal policy-maker with $\theta=\theta^{*}$, equation (7), can likewise be written

$$
\begin{aligned}
\theta^{*} & =A\left(y\left(\theta^{*}\right)\right)+c\left(y\left(\theta^{*}\right)-\theta^{*}\right) \\
& =\Phi\left(\sqrt{\alpha}\left(x^{*}-y^{*}\right)\right)+c\left(y^{*}-\theta^{*}\right) \\
& =\Phi(-\sqrt{\alpha} \gamma)+c\left(x^{*}+\gamma-\theta^{*}\right)
\end{aligned}
$$

Hence combining equations (38) and (39) gives

$$
\Phi\left(\sqrt{\alpha}\left(\theta^{*}-x^{*}\right)\right)=\frac{p}{1-p} \theta^{*}
$$

Now eliminating the common term $\Phi\left(\sqrt{\alpha}\left(\theta^{*}-x^{*}\right)\right)$ between (37) and (40) gives the simple relationship

$$
m\left(x^{*}\right)=\frac{\theta^{*}}{1-p}
$$

This is the same as equation (26) from the main text. If we let $k^{*}=\Phi\left(\sqrt{\alpha}\left(x^{*}-\theta^{*}\right)\right)$ and $k^{* *}=\Phi\left(\sqrt{\alpha}\left(x^{*}-\theta^{* *}\right)\right)$ then we can also write the policy-maker's indifference condition

$$
\theta^{*}=k^{* *}+c\left(\theta^{* *}-\theta^{*}\right)
$$

and likewise we also have

$$
m\left(x^{*}\right)=1+c\left(\theta^{* *}-\theta^{*}\right)+\left(k^{* *}-k^{*}\right)
$$

\section{Proof of Proposition 2.}

The aggregate action is the proportion of individuals with signals below $x^{*}$, that is $A(y(\theta))=l\left(x^{*}, \theta\right)=$ $\Phi\left(\sqrt{\alpha}\left(x^{*}-y(\theta)\right)\right)$. Let $k^{*}:=\bar{k}\left(x^{*}\right)=\Phi\left(\sqrt{\alpha}\left(x^{*}-\theta^{*}\right)\right)$ and $k^{* *}:=\underline{k}\left(x^{*}\right)=\Phi\left(\sqrt{\alpha}\left(x^{*}-\theta^{* *}\right)\right)$ denote the corresponding critical proportions. Since $G^{*}(k):=G\left(k \mid x^{*}\right)$, applying Proposition 1 we immediately have

$$
G^{*}(k)= \begin{cases}\frac{1}{m\left(x^{*}\right)} k & k \in\left[0, k^{* *}\right) \\ \frac{1}{m\left(x^{*}\right)} k^{* *}+J\left(x^{*}\right) & k \in\left[k^{* *}, k^{*}\right) \\ \frac{1}{m\left(x^{*}\right)}\left(k+k^{* *}-k^{*}\right)+J\left(x^{*}\right) & k \in\left[k^{*}, 1\right]\end{cases}
$$

where

$$
J\left(x^{*}\right)=\frac{\sqrt{\alpha} \phi\left(\sqrt{\alpha}\left(x^{*}-\theta^{* *}\right)\right)}{m\left(x^{*}\right)}\left(\theta^{* *}-\theta^{*}\right)=\frac{c}{m\left(x^{*}\right)}\left(\theta^{* *}-\theta^{*}\right)
$$


where the second equality uses the properties of the signaling policy $y^{*}=\theta^{* *}=x^{*}+\gamma$ and where the coefficient $\gamma$ solves $\sqrt{\alpha} \phi(\sqrt{\alpha} \gamma)=c$. From equation (41) above the marginal satisfies $m\left(x^{*}\right)=\theta^{*} /(1-p)$, so we can immediately simplify (44) to

$$
G^{*}(k)= \begin{cases}\frac{1-p}{\theta^{*}} k & k \in\left[0, k^{* *}\right) \\ \frac{1-p}{\theta^{*}}\left(k^{* *}+c\left(\theta^{* *}-\theta^{*}\right)\right) & k \in\left[k^{* *}, k^{*}\right) \\ \frac{1-p}{\theta^{*}}\left(k+k^{* *}-k^{*}+c\left(\theta^{* *}-\theta^{*}\right)\right) & k \in\left[k^{*}, 1\right]\end{cases}
$$

We can tidy this up by writing the policy-maker's indifference condition $\theta^{*}=k^{* *}+c\left(\theta^{* *}-\theta^{*}\right)$, as in equation (42) above, and by writing the alternative expression for the marginal $m\left(x^{*}\right)=1+c\left(\theta^{* *}-\theta^{*}\right)+\left(k^{* *}-k^{*}\right)$, as in equation (43) above, to get

$$
G^{*}(k)= \begin{cases}\frac{1-p}{\theta^{*}} k & k \in\left[0, k^{* *}\right) \\ 1-p & k \in\left[k^{* *}, k^{*}\right) \\ \frac{1-p}{\theta^{*}}(k-1)+1 & k \in\left[k^{*}, 1\right]\end{cases}
$$

which is the stated formula.

\section{Proof of Proposition 3.}

To begin with, observe that, independent of the signal precision $\alpha$, the only proportions $k$ that attract positive support are (i) $k \in\left[0, k^{* *}\right]$ and (ii) $k \in\left[k^{*}, 1\right]$. The intervening middle interval $\left(k^{* *}, k^{*}\right)$ has zero probability. In particular, for any signal precision $\alpha$ we have

$$
\operatorname{Prob}\left[0 \leq A(y(\theta)) \leq k^{* *}\right]=G^{*}\left(k^{* *}\right)=1-p
$$

and similarly

$$
\operatorname{Prob}\left[k^{*} \leq A(y(\theta)) \leq 1\right]=1-G^{*}\left(k^{* *}\right)=p
$$

All that varies with the signal precision are the critical proportions $k^{* *}$ and $k^{*}$. Recall the formula (29) for $k^{* *}$ in the main text, namely

$$
k^{* *}=\Phi(-\sqrt{\alpha} \gamma)=\Phi\left(-\sqrt{2 \log \left(\frac{\sqrt{\alpha}}{c} \phi(0)\right)}\right)
$$

So in the limit

$$
\lim _{\alpha \rightarrow+\infty} k^{* *}=\lim _{\alpha \rightarrow+\infty} \Phi\left(-\sqrt{2 \log \left(\frac{\sqrt{\alpha}}{c} \phi(0)\right)}\right)=0^{+}
$$

Hence the lower support collapses to the extreme lower bound, $k \in\left[0, k^{* *}\right] \rightarrow\{0\}$, and we have

$$
\lim _{\alpha \rightarrow+\infty} \operatorname{Prob}\left[0 \leq A(y(\theta)) \leq k^{* *}\right]=\operatorname{Prob}[A(y(\theta))=0]=1-p
$$

Similarly, from (28) above the upper critical point $k^{*}$ can be written

$$
k^{*}=1-\frac{p}{1-p} \theta^{*}
$$

And on applying Lemma 1 we have

$$
\lim _{\alpha \rightarrow+\infty} k^{*}=1-\frac{p}{1-p} \lim _{\alpha \rightarrow+\infty} \theta^{*}=1^{-}
$$

Hence the upper support collapses to the extreme upper bound, $k \in\left[k^{*}, 1\right] \rightarrow\{1\}$, and we have

$$
\lim _{\alpha \rightarrow+\infty} \operatorname{Prob}\left[k^{*} \leq A(y(\theta)) \leq 1\right]=\operatorname{Prob}[A(y(\theta))=1]=p
$$

So in the limit as the signal precision $\alpha \rightarrow+\infty$ the beliefs of the marginal individual approach the discrete binominal distribution on the extreme outcomes $\{0,1\}$ with probabilities $\{1-p, p\}$. 


\section{References}

Angeletos, George-Marios and Iván Werning, "Crises and Prices: Information Aggregation, Multiplicity and Volatility," American Economic Review, 2006, 96 (5), 1720-1736.

_, Christian Hellwig, and Alessandro Pavan, "Signaling in a Global Game: Coordination and Policy Traps," Journal of Political Economy, 2006, 114 (3), 452-484.

_ , _, and _ , "Dynamic Global Games of Regime Change: Learning, Multiplicity, and the Timing of Attacks," Econometrica, 2007, 75 (3), 711-756.

Calvo, Guillermo A., "Servicing the Public Debt: The Role of Expectations," American Economic Review, September 1988, 78 (4), 647-671.

Carlsson, Hans and Eric van Damme, "Global Games and Equilibrium Selection," Econometrica, 1993, 61 (5), 989-1018.

Chamley, Christophe P., "Coordinating Regime Switches," Quarterly Journal of Economics, August 1999, 114 (3), 869-905.

Cole, Harold L. and Timothy J. Kehoe, "Self-Fulfilling Debt Crises," Review of Economic Studies, January 2000, 67 (1), 91-116.

Corsetti, Giancarlo, Amil Dasgupta, Stephen Morris, and Hyun Song Shin, "Does One Soros Make a Difference? A Theory of Currency Crises with Large and Small Traders," Review of Economic Studies, 2004, 71 (1), 87-113.

Dasgupta, Amil, "Coordination and Delay in Global Games," Journal of Economic Theory, 2007, 134, 195-225.

Diamond, Douglas W. and Philip H. Dybvig, "Bank Runs, Deposit Insurance, and Liquidity," Journal of Political Economy, June 1983, 91 (3), 401-419.

Edmond, Chris, "Information Manipulation, Coordination, and Regime Change," Review of Economic Studies, October 2013, 80 (4), 1422-1458.

Goldstein, Itay and Ady Pauzner, "Demand Deposit Contracts and the Probability of Bank Runs," Journal of Finance, June 2005, 60 (3), 1293-1327.

Hellwig, Christian, "Public Information, Private Information, and the Multiplicity of Equilibria in Coordination Games," Journal of Economic Theory, 2002, 107, 191-222.

_, Arijit Mukherji, and Aleh Tsyvinski, "Self-Fulfilling Currency Crises: The Role of Interest Rates," American Economic Review, December 2006, 96 (5), 1769-1787.

Katz, Michael L. and Carl Shapiro, "Technology Adoption in the Presence of Network Externalities," Journal of Political Economy, August 1986, 94 (4), 822-841.

Keynes, John Maynard, A Treatise on Probability, Macmillan, 1921. 
Morris, Stephen and Hyun Song Shin, "Unique Equilibrium in a Model of Self-Fulfilling Currency Attacks," American Economic Review, 1998, 88 (3), 587-597.

_ and _ , "Rethinking Multiple Equilibria in Macroeconomic Modeling," in Ben S. Bernanke and Kenneth Rogoff, eds., NBER Macroeconomics Annual, MIT Press 2000, pp. 139-161.

_ and _, "Global Games: Theory and Applications," in Mathias Dewatripont, Lars Peter Hansen, and Stephen J. Turnovsky, eds., Advances in Economics and Econometrics: Theory and Applications, Cambridge University Press 2003.

_ and _ , "Common Belief Foundations of Global Games," 2007. Princeton University working paper.

Obstfeld, Maurice, "Rational and Self-Fulfilling Balance-of-Payments Crises," American Economic Review, 1986, 76 (1), 72-81.

_ , "Models of Currency Crises with Self-Fulfilling Features," European Economic Review, 1996, 40 (3-5), 1037-1047.

Rochet, Jean-Charles and Xavier Vives, "Coordination Failures and the Lender of Last Resort: Was Bagehot Right after All?," Journal of the European Economic Association, December 2004, 2 (6), 1116-1147.

Stigler, Stephen M., The History of Statistics: The Measurement of Uncertainty before 1900, Harvard University Press, 1986.

Vives, Xavier, "Complementarities and Games: New Developments," Journal of Economic Literature, June 2005, 43 (2), 437-479. 